\title{
Efficacy of combining intravitreal injections of ranibizumab with micropulse diode laser versus intravitreal injections of ranibizumab alone in diabetic macular edema (ReCaLL): a single center, randomised, controlled, non-inferiority clinical trial
}

\author{
Olga Furashova ${ }^{1 *}$ iD, Patrick Strassburger ${ }^{1}$, Klio Ai Becker and Katrin Engelmann ${ }^{1}$
}

\begin{abstract}
Background: To evaluate if a combination therapy with micropulse diode laser (MPL) shows non-inferiority on visual acuity (BCVA) within 12 months in comparison to standard therapy, i.e. intravitreal injection of ranibizumab alone.

Setting: Institutional. Prospective randomized single-center trial.

Methods: Patients with diabetic macular edema (DME) received three intravitreal injections of $0.5 \mathrm{mg}$ ranibizumab during the upload phase and were then randomised 1:1 to receive either the same dosage of ranibizumab $(0.5 \mathrm{mg})$ injections pro re nata alone (IVOM-Group; $n=9$ ), or with two additional treatments with micropulse diode laser (IVOM+Laser-Group; $n=10$ ). The primary endpoint was change in BCVA after 12 months. Secondary endpoints were change in central macular thickness and overall number of ranibizumab injections.

Results: BCVA increased significantly in both groups (IVOM: + 5.86, $p<0.001$; IVOM+Laser: +9.30; $p<0.001$ ) with corresponding decrease in central macular thickness (IVOM: - $105 \mu \mathrm{m}, p<0.01$; IVOM+Laser: $-125 \mu \mathrm{m} ; \mathrm{p}<0.01$ ). Patients with additional laser treatment had better visual improvement (group comparison $p=0.075$ ) and needed fewer ranibizumab injections (cumulative proportion of injections 9.68 versus 7.46 in IVOM-Group and IVOM+LaserGroup, respectively).
\end{abstract}

Conclusion: Non-inferiority of combination therapy in comparison to standard therapy alone could be demonstrated. Patients with additional laser therapy needed fewer ranibizumab injections.

Trial registration: Registered 10 February 2014 on ClinicalTrials.gov; NCT02059772.

Keywords: Diabetic macular edema, Ranibizumab, VEGF inhibitor, Intravitreal injection, Micropulse diode laser

\footnotetext{
* Correspondence: o.furashova@skc.de

'Department of Ophthalmology, Klinikum Chemnitz gGmbH,

Flemmingstrasse 2, 09116 Chemnitz, Germany

Full list of author information is available at the end of the article
}

(c) The Author(s). 2020 Open Access This article is licensed under a Creative Commons Attribution 4.0 International License, which permits use, sharing, adaptation, distribution and reproduction in any medium or format, as long as you give appropriate credit to the original author(s) and the source, provide a link to the Creative Commons licence, and indicate if changes were made. The images or other third party material in this article are included in the article's Creative Commons licence, unless indicated otherwise in a credit line to the material. If material is not included in the article's Creative Commons licence and your intended use is not permitted by statutory regulation or exceeds the permitted use, you will need to obtain permission directly from the copyright holder. To view a copy of this licence, visit http://creativecommons.org/licenses/by/4.0/ The Creative Commons Public Domain Dedication waiver (http://creativecommons.org/publicdomain/zero/1.0/) applies to the data made available in this article, unless otherwise stated in a credit line to the data. 


\section{Brief summary}

Combined treatment of diabetic macular edema with ranibizumab and micropulse diode laser is non inferior compared to standard therapy with ranibizumab alone. Patient with additional laser therapy needed fewer ranibizumab injections.

\section{Background}

Diabetic retinopathy (DRP) and diabetic macular edema (DME) are diseases of the retina that are caused by complications of diabetes mellitus. DRP is the third most frequently reason for visual loss in industrialized countries $[1,2]$. DME is caused by leaking macular capillaries and is the most common cause of visual loss in both proliferative and non-proliferative DRP.

Focal/grid macular laser photocoagulation is still sometimes used for therapy of vision-threatening DME $[3,4]$. However, it does have considerable disadvantages because of its destructive nature [5].

In contrast, micropulse diode laser therapy (MPL) causes less damage to photoreceptors and retinal pigment epithelium cells [6]. Moreover, there is evidence for an additional positive effect of high wavelength (810 $\mathrm{nm}$ ) of the diode laser supporting the recovery of cells especially those of the retinal pigment epithelium [7].

In 2011 the vascular endothelial growth factor (VEGF) inhibitor ranibizumab (Lucentis ${ }^{\circ}$, Novartis Pharma) was approved and recommended for the treatment of DME. Most of the patients treated with ranibizumab experience a qualitative as well as quantitative improvement of visual function within a few days after treatment already $[8,9]$. However, the effect is only short-lasting thus requiring multiple follow-up injections, usually 7 to 9 in the first year of treatment $[9,10]$. Moreover, up to now it remains open whether multiple injections may cause damage to photoreceptors and other parts of the retina $[11,12]$.

In several studies on patients with DME it was shown that the effect of MPL to that of the "standard" laser (gold standard) is at least comparable [13-16]. In clinical practice a two-time treatment with the diode laser is routinely performed in order to provide a long-lasting improvement of the visual function. Treatment with the VEGF-inhibitor ranibizumab leads to rapid improvement of DME. In contrast, the laser treatment is much slower and shows a weaker effect but the effect is more sustainable. Thus, the disadvantages of laser treatment might be circumvented by an initial combination therapy with ranibizumab.

In the ReCaLL study micropulse diode laser therapy was combined with the German Ophthalmological Society (DOG)-recommended first-line therapy, i.e. the intravitreal injection of the VEGF-inhibitor ranibizumab in dosage of $0.5 \mathrm{mg}$. The aim of the study was to investigate whether a combination therapy of micropulse diode laser treatment and intravitreal injection of the VEGFinhibitor ranibizumab may improve the visual function more efficaciously than anti-VEGF injection alone and additionally minimize potential complications by reduction of injection frequency.

\section{Methods}

ReCaLL (NCT02059772) was a single-center phase 4, prospective, open-label, randomized controlled study in Germany between April 2014 and December 2016. The study received approval from the independent ethics committee and was conducted in accordance with the Declaration of Helsinki and International Conference on Harmonization Good Clinical Practice guidelines. The study adheres to CONSORT guidelines. All participants provided written informed consent prior to inclusion in the study.

Patients included in this study met the following criteria: 1) diagnosed with non-ischemic DME; 2) BCVA between 35 and 89 on ETDRS charts or central retinal thickness > $300 \mu \mathrm{m}$ as determined by spectral domain optical coherence tomography (SD-OCT). The exclusion criteria were: 1) severe ischemic maculopathy; 2) active neovascularization of iris or retina; 3) history of intravitreal injection of VEGF-inhibitor or steroids within the last 3 months; 4) pathologies of the anterior segment with reduced visual acuity (e.g. corneal opacification, advanced cataract); 5) other ocular pathologies with reduced visual acuity (e.g. central scars, age related macular degeneration, retinal vascular occlusion in medical history); 6) active or suspected ocular or periocular infections; 7) intraocular surgery or laser therapy within the preceding 6 months; 8) systemic steroid therapy within the last 3 months; 9) HbAlc greater than $10 \%$ or blood pressure above $170 / 110 \mathrm{mmHg}$.

After the first 3 monthly intravitreal injections of 0.5 $\mathrm{mg}$ ranibizumab (Lucentis ${ }^{\circ}$ ), subjects were randomised 1:1 to receive either standard treatment with ranibizumab pro re nata (IVOM-Group) or standard treatment plus two additional applications of micropulse diode laser (IVOM+Laser-Group) at Visits 5 and 6. Ranibizumab was injected in the same dosage of $0.5 \mathrm{mg}$ in both groups. Visits were scheduled every 4 weeks until primary endpoint at 12 months. If retreatment criteria were met, follow-up injections of ranibizumab were given in both groups every 4 weeks until stability of BCVA was reached again. In IVOM+Laser-Group ranibizumab injections at Visits 5 and 6 were administered at least 30 min after laser photocoagulation. Physicians and technicians handling the patients were not masked about the grouping of the patients.

Table 1 demonstrates criteria for continuing or stopping ranibizumab treatment according to pro re nata (PRN). The primary endpoint was the mean change in BCVA over 12 months on ETDRS charts. Secondary endpoints were the mean change in CMT over 12 
Table 1 Re-treatment and stopping treatment criteria for pro re nata (PRN) regimen used in the study

\begin{tabular}{ll}
\hline Criteria for re-treatment & Criteria for stopping treatment \\
\hline visual improvement & irreversible changes of central macula (i.e. atrophy, ischemia) without the perspective for visual improvement \\
decrease in CMT $\geq 10 \%{ }^{\mathrm{a}}$ & BCVA $<35$ as measured by ETDRS charts ${ }^{\mathrm{b}}$ \\
& BCVA of 100 on ETDRS charts \\
& lack of fluid in the area of the fovea
\end{tabular}

CMT central macular thickness; BCVA best corrected visual acuity; ETDRS Early Treatment Diabetic Retinopathy Study;

a thickness evaluation under consideration of cysts and vitreoretinal membrane structures

${ }^{b}$ with the exception of visual impairment due to other causes such as vitreous hemorrhage

months determined by SD-OCT, and the overall number of treatments with ranibizumab.

Figure 1 shows the trial flowchart with time frames of the present study.

\section{Laser treatment protocol}

Laser treatment was performed with OcuLight SLx, Symphony Tri-Mode, IRIDEX Corp., Mountain View, CA, USA, at a wavelength of $810 \mathrm{~nm}$ using the magnifier Volk Optical Inc., Mentor, OH, USA. Treatment was performed as confluent laser photocoagulation of the macula without area of the fovea with a duty cycle of $15 \%$, at doubled threshold energy and an exposure time of $200 \mathrm{msec}$.

\section{Statistical analysis}

Approximately 50 individuals were to be enrolled in the study, i.e. 25 participants per study group. This sample size included a drop-out rate of $15 \%$ and had $90 \%$ power to detect a difference in means of $20 \%$, assuming a standard deviation (SD) of 0.07 in a two-group t-test at 0.05 level of significance (one-sided). However, enrollment for this study was prematurely stopped after inclusion of 25 patients as the recruitment was substantially delayed. This was deemed to have no impact on the study outcome due to the exploratory character. All statistical analyses were performed on an explorative basis. Comparisons with no further statement of direction were tested at the 2-sided 5\% significance level. Primary target analysis for non-inferiority was based on a onesided 5\% significance level referring to the stated direction in the statistical hypotheses. Analyses of continuous variables (BCVA, CMT) were performed by using Student's t-test for independent variables (inter-group comparison of means or change of primary and secondary target variables) as well as Student's t-test for dependent or related samples (intra-group comparison of mean at selected study visits). Visual acuity measurements were analyzed using ETDRS charts with final conversion to Snellen charts. IBM SPSS Statistics, version 23.0.0.0 for Windows (IBM, Armonk, NY, USA) was used to perform the analysis.

Efficacy endpoints were analyzed for the intent-totreat set (ITT; all randomized participants) and for the per-protocol set (PPS; subjects who had complete data of primary and secondary target variables at the first and last visit, with no major protocol deviations thought to impact on the efficacy conclusions of the trial).

\section{Results}

Overall, 17 of 25 included patients completed the study. For participants flow chart please see Table 2.

Demographic and baseline characteristics of all randomized subjects were not statistically significantly different and are given in Table 3.

Tables 4 and 5 show the change in BCVA and CMT over the whole study period in both groups.

\section{BCVA}

BCVA increased statistically significant in both treatment groups till month 12 . No significant differences could be found in BCVA change between the two groups at any time (Fig. 2). Notably, a tendency was observed for a greater improvement in IVOM+Laser-Group than in IVOM-Group between baseline and end of treatment when considering the ITT ( $p$-value for comparison of both groups at end of treatment 0.075 ), see Table 4.

\section{CMT}

CMT decreased significantly in each treatment group over the study course. The effect of the treatment on CMT seems to be more prominent within the upload phase than later on and laser treatment seems not to contribute to this effect. Inter-group comparison of mean absolute CMT values as well as changes in CMT revealed no statistically significant difference between the groups (Fig. 2).

\section{Number of intravitreal injections}

Subjects in IVOM+Laser-Group received less injections of ranibizumab than subjects in IVOM-Group (PPS: on average 9 injections per patient in IVOM-Group and 7.5 in IVOM+ Laser-Group over a period of 12 months), see Fig. 3.

\section{Safety analysis}

There have been 3 cases of corneal erosion and 1 case of conjunctival inflammation after ranibizumab injection. No ocular adverse events have been observed after laser treatment. 


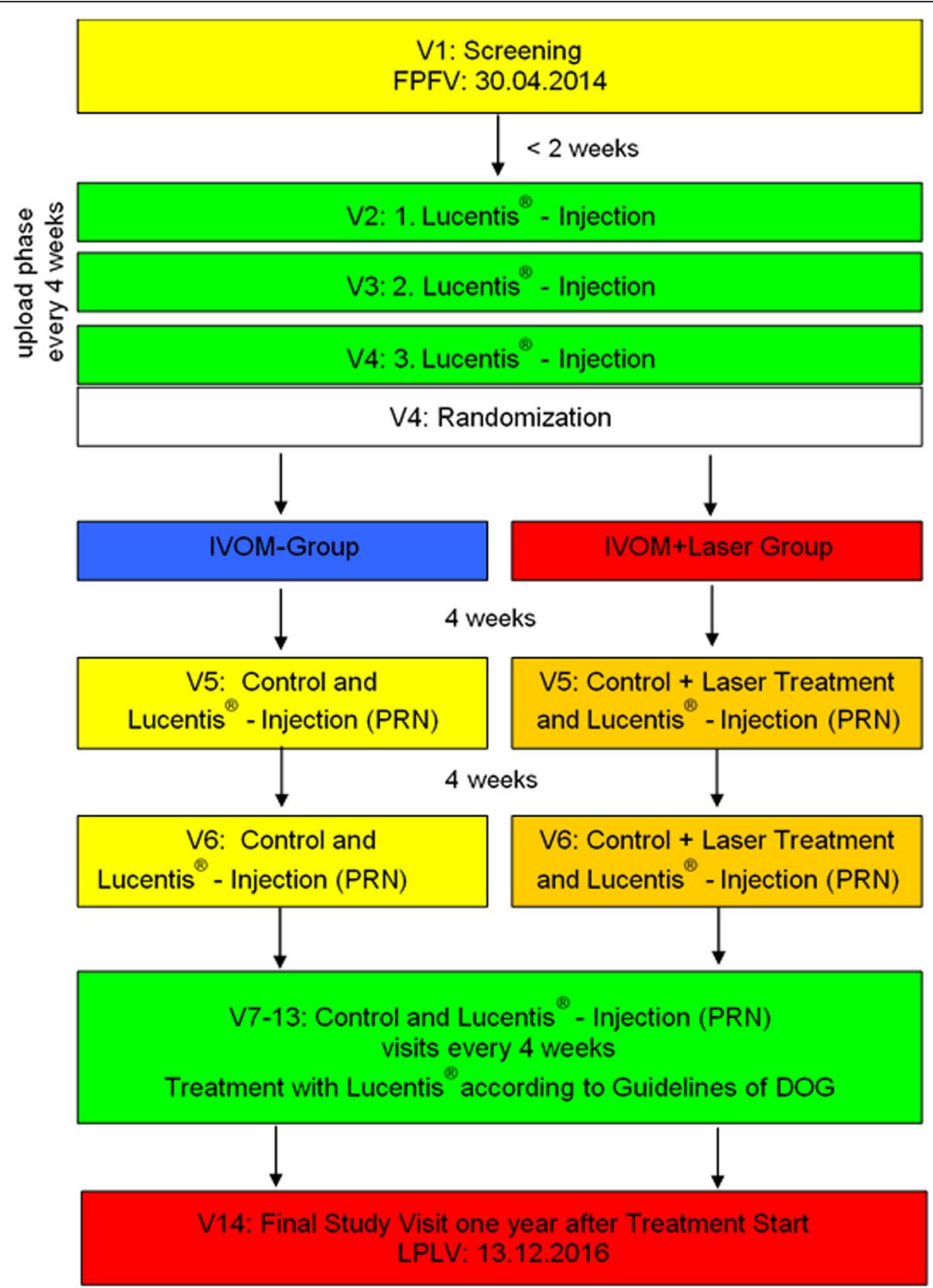

Fig. 1 Trial flowchart. FPFV - first patient's first visit; LPLV - last patient's last visit; IVOM - intravitreal injection; PRN - pro re nata; DOG Deutsche Ophthalmologische Gesellschaft (German Ophthalmology Society)

During the course of the study, three serious adverse events were documented. Two of them (apoplexy and myocardial infarction) were assessed as related to the study medication ranibizumab and for another one (asystole) relationship to the study medication could not be ruled out.

\section{Discussion}

The present study showed non-inferiority of combined micropulse diode laser treatment with intravitreal injections of ranibizumab compared to ranibizumab injections alone in patients with DME.

Micropulse diode laser (MPL) has been shown to be more effective in improving morphology and function in patients with DME compared to conventional laser treatment [17]. Furthermore, MPL is known as a tissuesparing laser treatment modality without laser-induced retinal damage. Chhablani et al. found significantly better retinal sensitivity parameters after subthreshold micropulse laser treatment compared to convetional 


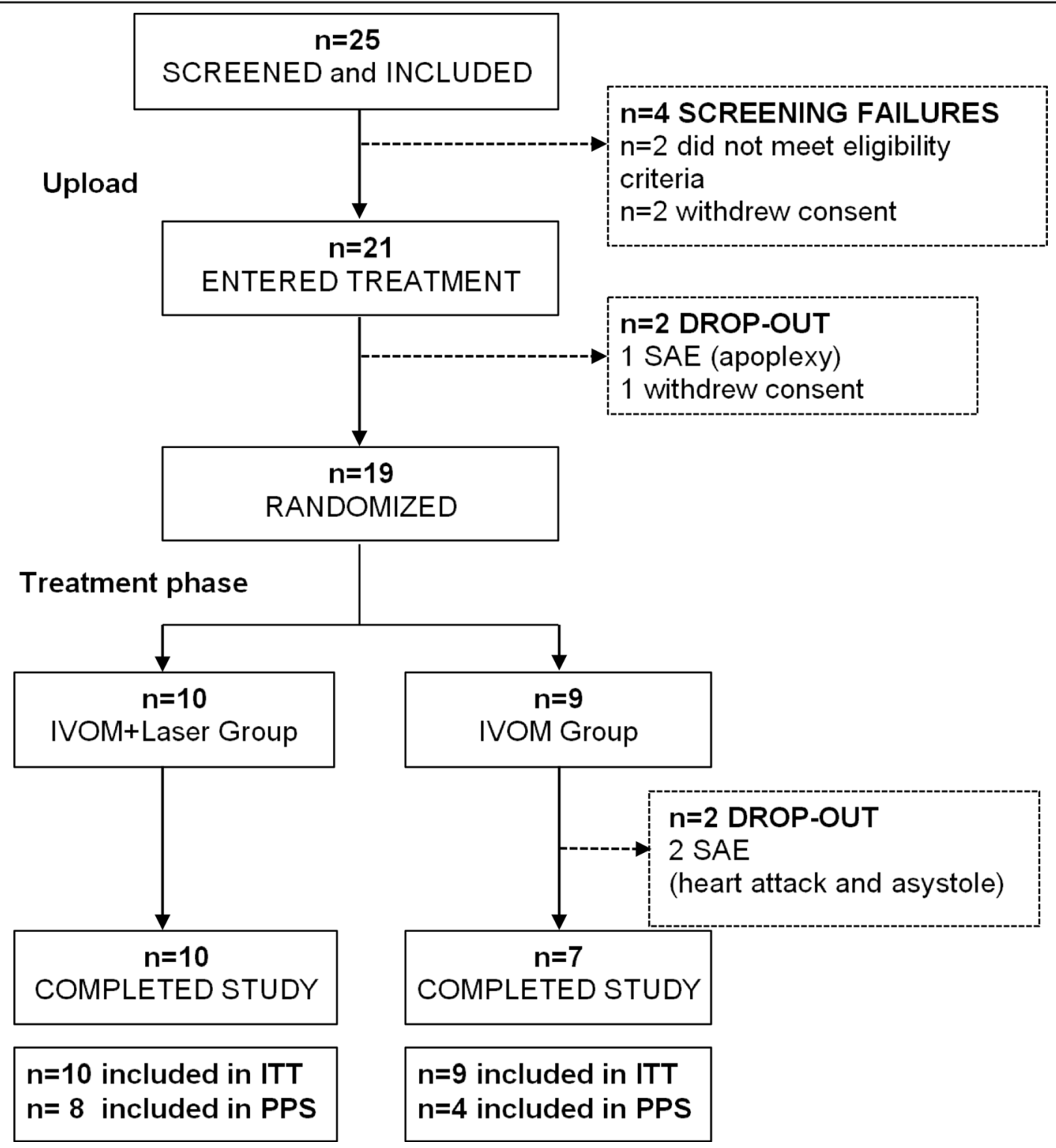

ITT intent-to-treat set (all randomized subjects); PPS per-protocol set (subjects with complete data of primary and secondary target variables at the first and last visit, with no major protocol deviations); SAE serious adverse event

continuous laser wave in DME patients [18]. A recent work of Midena et al. demonstrated also an additional anti-inflammatory effect of MDL [19].

Inagaki et al. showed recently, that combining antiVEGF injections and minimally invasive laser treatment in DME results in good anatomical and functional improvement at 12 months [20]. While the visual gain in the study of Inagaki et al. was slightly worse (5.9 ETDRS letters), than in previous prospective studies for antiVEGF therapy alone (6.6-10.3 EDTRS letters in REVE $\mathrm{Al}$, RESTORE and RESOLVE), the mean number of injections was as low as 3.612 months after treatment.
In our study, we also observed a statistically significant improvement in BCVA after 12 months with a mean visual gain of 5.25 ETDRS letters in the IVOM-Group and 9.50 ETDRS letters in the IVOM+Laser-Group. Interestingly, the change in BCVA between the end of upload phase (Visit 5) and the end of treatment did not reach significance for IVOM-Group but for IVOM+LaserGroup indicating that the laser treatment exhibits an additional effect (Table 4).

Liegl et al. observed a similar BCVA improvement in DME patients treated either with ranibizumab alone or in combination with navigated laser photocoagulation 
Table 3 Demographics and baseline characteristics (ITT, $N=19)$

\begin{tabular}{|c|c|c|c|c|c|}
\hline Variable & & $\begin{array}{l}\text { IVOM-Group } \\
(\mathrm{N}=9)\end{array}$ & IVOM + Laser-Group $(\mathrm{N}=10)$ & $\begin{array}{l}\text { Total } \\
(\mathrm{N}=19)\end{array}$ & $p$-value ${ }^{a}$ \\
\hline Age (years) & Mean \pm SD & $70.78 \pm 8.96$ & $70.70 \pm 7.60$ & $70.74 \pm 8.03$ & 0.984 \\
\hline \multicolumn{6}{|l|}{ Gender } \\
\hline Male & $N(\%)$ & $6(66.7 \%)$ & $8(80.0 \%)$ & $14(73.7 \%)$ & \\
\hline Female & $N(\%)$ & $3(33.3 \%)$ & $2(20.0 \%)$ & $5(26.3 \%)$ & \\
\hline \multicolumn{6}{|l|}{ Race } \\
\hline White & $N(\%)$ & $9(100 \%)$ & $10(100 \%)$ & $19(100 \%)$ & \\
\hline Weight (kg) & Mean \pm SD & $92.83 \pm 8.15$ & $83.90 \pm 12.92$ & $88.13 \pm 11.58$ & 0.093 \\
\hline Height (cm) & Mean \pm SD & $173 \pm 12$ & $173 \pm 8$ & $173 \pm 10$ & 0.860 \\
\hline $\mathrm{BMI}\left(\mathrm{kg} / \mathrm{m}^{2}\right)$ & Mean \pm SD & $31.13 \pm 3.71$ & $28.04 \pm 2.38$ & $29.51 \pm 3.38$ & 0.043 \\
\hline Diastolic blood pressure (mmHg) & Mean \pm SD & $82.13 \pm 13.16$ & $80.40 \pm 9.38$ & $81.17 \pm 10.90$ & 0.750 \\
\hline Systolic blood pressure (mmHg) & Mean \pm SD & $147.0 \pm 14.22$ & $151.1 \pm 10.91$ & $149.3 \pm 12.28$ & 0.498 \\
\hline $\mathrm{HbA1c}(\%)$ & Mean \pm SD & $7.54 \pm 1.51$ & $6.80 \pm 0.85$ & $7.19 \pm 1.26$ & 0.274 \\
\hline \multicolumn{6}{|l|}{ Study eye } \\
\hline Left & N (\%) & 7 (77.8\%) & $5(50 \%)$ & 12 (63.2\%) & \\
\hline Right & N (\%) & 2 (22.2\%) & $5(50 \%)$ & 7 (36.8\%) & \\
\hline BCVA & Mean & $20 / 120$ & 20/95 & $20 / 110$ & 0.478 \\
\hline CMT $(\mu \mathrm{m})$ & Mean \pm SD & $485 \pm 170$ & $434 \pm 118$ & $458 \pm 143$ & 0.456 \\
\hline
\end{tabular}

$I T T$ intent-to-treat set (all randomized subjects); BMI body mass index; BCVA best corrected visual acuity; CMT central macular thickness;

a comparisons between groups were done using Student's t-test for independent variables

(8.41 vs. 6.31 ETDRS letters, $p=0.258$ ) [21]. However, in the group of combined treatment with ranibizumab injections and navigated laser photocoagulation, there were significantly less anti-VEGF injections required during the 12 months of follow up time (3.9 injections in the combination group vs. 6.9 in the injection group).

A recent retrospective study of Moiseiev et al. could also show a significant reduction in the burden of antiVEGF injections when combining with MPL for DME treatment [22]. Kanar et al. demonstrated in a randomized clinical trial, that combining intravitreal aflibercept injections with subthreshold micropulse yellow laser for DME treatment results in fewer intravitreal injections and similar anatomic and functional outcome [23].
The results of our study confirm the findings of previous studies, showing fewer intravitreal injections of ranibizumab needed in the IVOM+Laser-Group compared to IVOM-Group (7.5 vs. 9.0, respectively). The mean number of intravitreal ranibizumab injections in our study was higher than in the cohorts of Liegl et al. and Inagaki et al. This might be explained by different PRN re-treatment criteria, timing of laser therapy as well as different patients' characteristics including diabetes status and other systemic conditions (e.g. arterial hypertension), influencing the course of DME.

Nowadays, follow-up visits for injection treatments take place every 4 weeks. Furthermore, according to German regulations, patients are obliged to visit their

Table 4 Intra-group and inter-group comparison of BCVA during the study period

\begin{tabular}{|c|c|c|c|c|}
\hline \multirow[t]{2}{*}{ Dataset } & & \multicolumn{3}{|c|}{ Change in BCVA (mean \pm SD) } \\
\hline & & Visit $1 \rightarrow$ Visit 5 & Visit $5 \rightarrow$ Visit 14 & Visit $1 \rightarrow$ Visit 14 \\
\hline \multirow[t]{3}{*}{$\overline{I T T}$} & IVOM & $4.11 \pm 6.31(p=0.087)$ & $2.86 \pm 5.08(p=0.187)$ & $5.86 \pm 1.86(p<0.001)$ \\
\hline & IVOM+Laser & $4.30 \pm 6.96(p=0.083)$ & $5.00 \pm 4.74(p=0.009)$ & $9.30 \pm 5.12(p<0.001)$ \\
\hline & IVOM vs. IVOM+Laser & $p=0.952$ & $p=0.387$ & $p=0.075$ \\
\hline \multirow[t]{3}{*}{ PPS } & IVOM & $5.00 \pm 6.88(p=0.242)$ & $0.25 \pm 5.12(p=0.928)$ & $5.25 \pm 2.06(p=0.015)$ \\
\hline & IVOM+Laser & $4.00 \pm 7.29(p=0.165)$ & $5.50 \pm 5.21(p=0.020)$ & $9.50 \pm 5.26(p=0.001)$ \\
\hline & IVOM vs. IVOM+Laser & $p=0.824$ & $p=0.129$ & $p=0.158$ \\
\hline
\end{tabular}

$I T$ intent-to-treat set (all randomized subjects); PPS per-protocol set (subjects with complete data of primary and secondary target variables at the first and last visit, with no major protocol deviations); $B C V A$ best corrected visual acuity;

Comparisons between groups were done using Student's t-test for dependent samples. BCVA changes are expressed in ETDRS charts for better statistical analysis 
Table 5 Intra-group and inter-group comparison of CMT during the treatment period

\begin{tabular}{|c|c|c|c|c|}
\hline \multirow{2}{*}{$\begin{array}{l}\text { Data } \\
\text { set }\end{array}$} & & \multicolumn{3}{|c|}{ Change in CMT ( $\mu$ m; mean $\pm S D)$} \\
\hline & & Visit $1 \rightarrow$ Visit 5 & Visit $5 \rightarrow$ Visit 14 & Visit $1 \rightarrow$ Visit 14 \\
\hline \multirow[t]{3}{*}{ ITT } & IVOM & $-90.33 \pm 114.65(p=0.046)$ & $20.43 \pm 79.61(p=0.523)$ & $-104.86 \pm 68.76(p=0.007)$ \\
\hline & IVOM+Laser & $-107.80 \pm 56.24(p<0.001)$ & $-16.70 \pm 62.96(p=0.423)$ & $-124.50 \pm 81.08(p=0.001)$ \\
\hline & IVOM vs. IVOM+Laser & $p=0.673$ & $p=0.299$ & $p=0.609$ \\
\hline \multirow[t]{3}{*}{ PPS } & IVOM & $-94.25 \pm 77.47(p=0.093)$ & $29.00 \pm 34.28(p=0.189)$ & $-65.25 \pm 67.57(p=0.149)$ \\
\hline & IVOM+aser & $-99.13 \pm 50.55(p=0.001)$ & $-18.25 \pm 71.21(p=0.492)$ & $-117.38 \pm 82.71(p=0.005)$ \\
\hline & IVOM vs. IVOM+Laser & $p=0.897$ & $p=0.245$ & $p=0.304$ \\
\hline
\end{tabular}

$I T T$ intent-to-treat set (all randomized subjects); PPS per-protocol set (subjects with complete data of primary and secondary target variables at the first and last visit, with no major protocol deviations); CMT central macular thickness;

Comparisons between groups were done using Student's t-test for dependent samples.

ophthalmologist 1-3 days after every injection, thus making at least 2 visits pro injection necessary. This requires enormous efforts by patients, ophthalmic surgeons and ophthalmologists.

The results of our study support previous findings showing non-inferiority of combined treatment of DME with anti-VEGF injections and micropulse diode laser versus anti-VEGF injections alone. We could not find any differences in anatomical and functional outcome in both groups after 1 year of treatment. Furthermore, additional micropulse tissue-saving laser treatment in DME seems to reduce the number of intravitreal injections, thus improving the quality of life of both ophthalmologists and their patients as well as reducing the economic burden.

It should be mentioned, that in IVOM+Laser-Group, the patients required a mean of 1.5 fewer injections but had two additional laser treatment visits. However, regarding the known risks of possible ocular and systemic complications of both treatment modalities as well as the costs of laser treatment compared to intravitreal injections, we believe, that combined therapy still has its economic benefits. Furthermore, our study represented the disease treatment course only over 12 months. It would be interesting to know, whether the patients in the IVOM+Laser-Group had further benefit of fewer injections after 12 months.

One major limitation of this Phase 4 study was the low sample size. This might have compromised the study results and their validation. It is well known, that the treatment outcome in DME is associated with baseline characteristics such as disease duration, HbA1c, blood pressure level, BCVA, CMT, etc. In our study, both treatment groups showed no statistically significant differences in the baseline parameters, but this might also have been compromised by the small sample size.

The study was planned to include 50 patients but finally only 19 were randomized in total and even only 12 could be included in the PPS group. High efforts were required to enroll an appropriate number of eligible and willing patients for the study in a reasonable timeframe. This was accompanied by lack of compliance by some of the study participants. But in the end, this mirrors the conditions of the patient population, i.e. elderly people with diabetes and a series of co-morbidities for whom it would be of great benefit to reduce the strenuous effort caused by frequent visits to the ophthalmologist and repeated treatments.
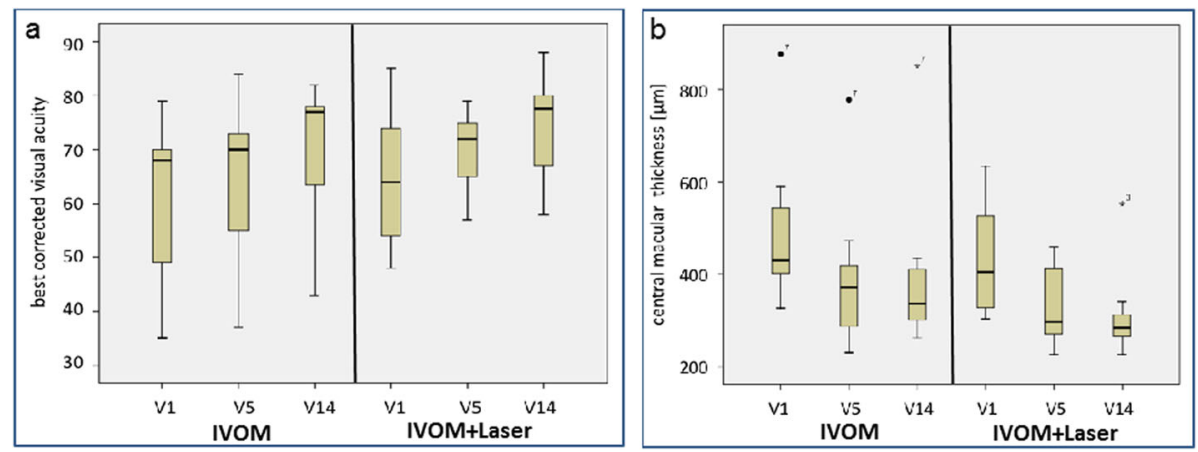

Fig. 2 a - Boxplot for BCVA (best corrected visual acuity) measured with ETDRS charts (ITT, $n=19$ ); no statistically significant difference could be observed between the groups at baseline (V1), after the upload phase (V5) and at the end of treatment (V14); BCVA values expressed in ETDRS charts; $\mathbf{b}$ - Boxplot for CMT (central macular thickness; $\mu \mathrm{m} ; \mathrm{ITT}, \mathrm{n}=19$ ); no statistically significant difference could be observed between the groups at baseline (V1), after the upload phase (V5) and at the end of treatment (V14) 


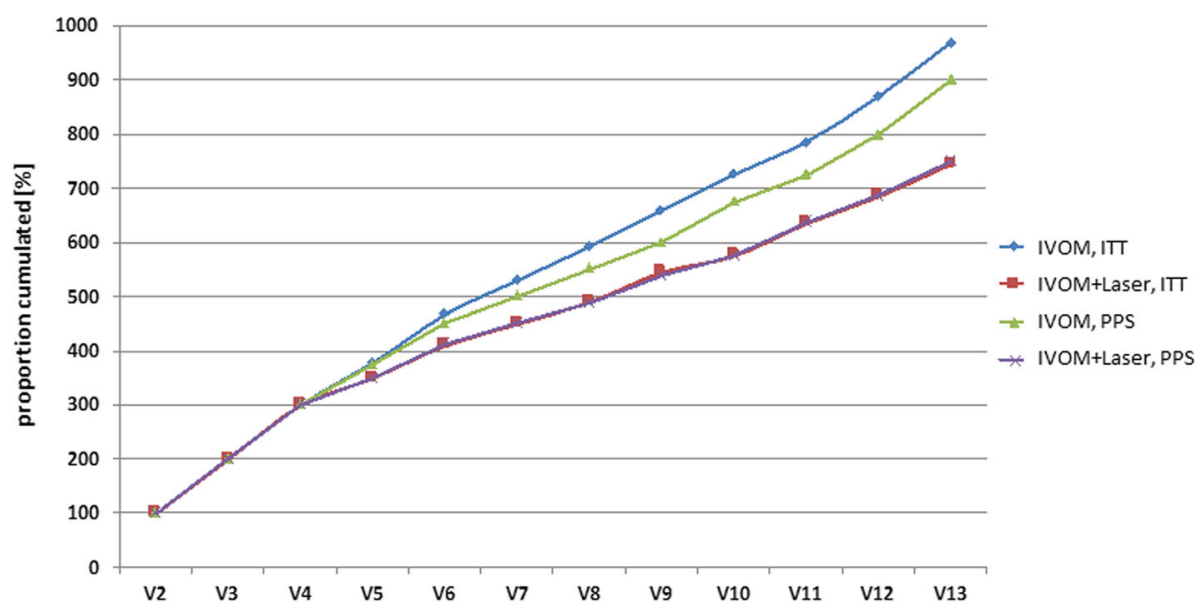

Fig. 3 Cumulative proportion (\%) of ranibizumab injections per visit. ITT - intent-to-treat set (all randomized subjects); PPS - per-protocol set (subjects with complete data of primary and secondary target variables at the first and last visit, with no major protocol deviations)

\section{Conclusion}

Our results support the findings of other study groups, who also observed a benefit of additional micropulse diode laser treatment in DME patients treated with ranibizumab. While the functional and anatomical results show noninferiority of combined treatment versus anti-VEGF injections alone, the injection frequency can be reduced suggesting better quality of life and economic benefit.

\section{Abbreviations}

BCVA: Best Corrected Visual Acuity; DME: Diabetic Macular Edema; IVOM: Intravitreal Injection; VEGF: Vascular Endothelial Growth Factor; DRP: Diabetic Retinopathy; MPL: Micropulse Laser; DOG: Deutsche Ophthalmologische Gesellschaft = German Ophthalmological Society; ETDR S: Early Treatment Diabetic Retinopathy Study; SD-OCT: Spectral Domain Optical Coherence Tomography; PRN: pro re nata; CMT: Central Macular Thickness; ITT: Intent-To-Treat Set (all randomized subjects); PPS: Per-Protocol Set (subjects with complete data of primary and secondary target variables at the first and last visit, with no major protocol deviations); SAE: Serious Adverse Event; SD: Standard Deviation

\section{Acknowledgements}

the authors thank Martin Strangfeld for statistical input and Antje Gasparic for medical writing support and editorial assistance.

\section{Authors' contributions}

OF: data acquisition; data interpretation and analysis; manuscript draft and revision; approval of the submitted version; agreement both to be personally accountable for the author's own contributions and to ensure that questions related to the accuracy or integrity of any part of the work, even ones in which the author was not personally involved, are appropriately investigated, resolved, and the resolution documented in the literature. PS: data acquisition; approval of the submitted version; agreement both to be personally accountable for the author's own contributions and to ensure that questions related to the accuracy or integrity of any part of the work, even ones in which the author was not personally involved, are appropriately investigated, resolved, and the resolution documented in the literature. KB: design of the work; data acquisition; approval of the submitted version; agreement both to be personally accountable for the author's own contributions and to ensure that questions related to the accuracy or integrity of any part of the work, even ones in which the author was not personally involved, are appropriately investigated, resolved, and the resolution documented in the literature. KE: design of the work; data interpretation and analysis; manuscript revision; approval of the submitted version; agreement both to be personally accountable for the author's own contributions and to ensure that questions related to the accuracy or integrity of any part of the work, even ones in which the author was not personally involved, are appropriately investigated, resolved, and the resolution documented in the literature. All authors have read and approved the manuscript

\section{Funding}

Conduct of the clinical study and preparation of the manuscript were financially supported by Novartis Pharma GmbH. GWT-TUD GmbH acted as sponsor of the study according to the requirements of the German Drug Law.

\section{Availability of data and materials}

The datasets during and/or analysed during the current study available from the corresponding author on reasonable request.

\section{Ethics approval and consent to participate}

The study received approval from the independent ethics committee and was conducted in accordance with the Declaration of Helsinki and International Conference on Harmonization Good Clinical Practice guidelines. The study adheres to CONSORT guidelines. All participants provided written informed consent prior to inclusion in the study. Ethics committee: Ethikkommission der Sächsischen Ladesärztekammer, Dresden; reference number EK-AMG-MO/3/13-1.

\section{Consent for publication}

Not applicable.

\section{Competing interests}

The authors declare that they have no competing interests.

\section{Author details}

${ }^{1}$ Department of Ophthalmology, Klinikum Chemnitz gGmbH, Flemmingstrasse 2, 09116 Chemnitz, Germany. ${ }^{2}$ Hessemer MVZ, Augenmedizin Darmstadt, Martinspfad 72, 64285 Darmstadt, Germany.

Received: 15 December 2019 Accepted: 20 July 2020

Published online: 29 July 2020

\section{References}

1. Cheung N, Mitchell P, et al. Diabetic retinopathy. Lancet. 2010 Jul 10; 376(9735):124-36.

2. Network DRCR, Elman MJ, Aiello LP, et al. Randomized trial evaluating ranibizumab plus prompt or deferred laser or triamcinolone plus prompt laser for diabetic macular edema. Ophthalmology. 2010;117(6):1064-77.

3. Early Treatment Diabetic Retinopathy Study research group. Photocoagulation for diabetic macular edema. Early Treatment Diabetic Retinopathy Study report number 1. Arch Ophthalmol. 1985;103(12):1796-806. 
4. Early Treatment Diabetic Retinopathy Study Group. Early photocoagulation for diabetic retinopathy. ETDRS report number 9. Ophthalmology. 1991;98(5 Suppl):766-85.

5. Cheung N, Mitchell P, Wong TY. Diabetic retinopathy. Lancet. 2010 Jul 10; 376(9735):124-36.

6. Scholz P, Altay L, Fauser S. A review of threshold micropulse laser for treatment of macular disorders. Adv Ther. 2017;34:1528-55.

7. Sramek C, Mackanos M, Spitler R, et al. Non-damaging retinal phototherapy: dynamic range of heat shock protein expression. Invest Ophthalmol Vis Sci. 2011;52(3):1780-7.

8. Massin P, Bandello F, Garweg JG, et al. Safety and efficacy of ranibizumab in diabetic macular edema (RESOLVE study): a 12-month, randomized, controlled, double-masked, multicenter phase II study. Diabetes Care. 2010 Nov;33(11):2399-405.

9. Mitchell P, Bandello F, Schmidt-Erfurth U, et al. The RESTORE study: ranibizumab monotherapy or combined with laser versus laser monotherapy for diabetic macular edema. Ophthalmology. 2011 Apr;118(4):615-25.

10. Elman MJ, Bressler NM, Qin H, et al. Diabetic retinopathy clinical research Network. Expanded 2-year follow-up of ranibizumab plus prompt or deferred laser or triamcinolone plus prompt laser for diabetic macular edema. Ophthalmology. 2011;118(4):609-14.

11. Quaggin SE. Turning a blind eye to anti-VEGF toxicities. J Clin Invest. 2012; 122(11):3849-51. https://doi.org/10.1172/JCl65509.

12. Kurihara T, Westenskow PD, Bravo $\mathrm{S}$, et al. Targeted deletion of Vegfa in adult mice induces vision loss. J Clin Invest. 2012 Nov;122(11):4213-7. https://doi.org/10.1172/JCl65157.

13. Figueira J, Khan J, Nunes $S$, et al. Prospective randomized controlled tria comparing subthreshold micropulse diode laser photocoagulation and conventional green laser for clinically significant diabetic macular oedema. Br J Ophthalmol. 2009;93(10):1341-4.

14. Lavinsky D, Cardillo JA, Melo LA Jr, et al. Randomized clinical trial evaluating mETDRS versus normal or high-density micropulse photocoagulation for diabetic macular edema. Invest Ophthalmol Vis Sci. 2011;52(7):4314-23.

15. Vujosevic S, Bottega $\mathrm{E}$, Casciano M, et al. Microperimetry and fundus autofluorescence in diabetic macular edema: subthreshold micropulse diode laser versus modified early treatment diabetic retinopathy study laser photocoagulation. Retina. 2010 Jun:30(6):908

16. Sivaprasad S, Dorin G. Subthreshold diode laser micropulse photocoagulation for the treatment of diabetic macular edema. Expert Rev Med Devices. 2012;9(2):189-97.

17. Scholz P, Altay L, Fauser S. A review of subthreshold micropulse laser for treatment of macular disorders. Adv Ther. 2017;34:1528-55. https://doi.org/ 10.1007/s12325-017-0559-y.

18. Chhablani J, Alshareef R, Kim DT, et al. Comparison of different settings for yellow subthreshold laser treatment in diabetic macular edema. BMC Ophthalmol. 2018;18:168. https://doi.org/10.1186/s12886-018-0841-z.

19. Midena E, Micera A, Frizziero L, et al. Sub-threshold micropulse laser treatment reduces infammatory biomarkers in aqueous humour of diabetic patients with macular edema. Sci Rep. 2019;9:10034. https://doi.org/10.1038/ s41598-019-46515-y.

20. Inagaki K, Hamada M, Ohkoshi K. Minimally invasive laser treatment combined with intravitreal injection of anti-vascular endothelial growth factor for diabetic macular oedema. Sci Rep. 2019;9:7585. https://doi.org/10. 1038/s41598-019-44130-5.

21. Liegl R, Langer J, Seidensticker F, et al. Comparative evaluation of combined navigated laser photocoagulation and Intravitreal Ranibizumab in the treatment of diabetic macular edema. PLoS One. 2014;12:e113981 doi: 10. 1371/ journal.pone.0113981.

22. Moisseiev E, Abbassi E, Thinda S, et al. Subthreshold micropulse laser reduces anti-VEGF injection burden in patients with diabetic macular edema. Eur J Ophthalmol. 2018;28:68-73. https://doi.org/10.5301/ejo. 5001000 .

23. Kanar HS, Arsan A, Altun A, et al. Can subthreshold micropulse yellow lase treatment change the anti-vascular endothelial growth factor algorithm in diabetic macular edema? A randomized clinical trial. Indian J Ophthalmol. 2020;68(1):145-51. https://doi.org/10.4103/ijo.IJO_350_19.

\section{Publisher's Note}

Springer Nature remains neutral with regard to jurisdictional claims in published maps and institutional affiliations.

Ready to submit your research? Choose BMC and benefit from:

- fast, convenient online submission

- thorough peer review by experienced researchers in your field

- rapid publication on acceptance

- support for research data, including large and complex data types

- gold Open Access which fosters wider collaboration and increased citations

- maximum visibility for your research: over $100 \mathrm{M}$ website views per year

At BMC, research is always in progress.

Learn more biomedcentral.com/submissions 